\title{
Isolation of Amidase-negative Mutants of Pseudomonas aeruginosa by a Positive Selection Method Using an Acetamide Analogue
}

\author{
By PATRICIA H. CLARKE AND RENEE TATA \\ Department of Biochemistry, University College London, \\ Gower Street, London, WCIE $6 B T$
}

\section{(Received 20 October 1972)}

Pseudomonas aeruginosa is resistant to many amino acid and purine and pyrimidine analogues which cause growth inhibition of Escherichia coli and this has precluded the use of such analogues for the isolation of mutants either resistant to feedback repression or derepressed for the synthesis of biosynthetic enzymes (Holloway, I969). Calhoun \& Jensen (1972) were able to increase the sensitivity of $P$. aeruginosa to certain metabolic analogues by changing the carbon source in the growth medium. $p$-Amino-phenylalanine and $\beta$-2-thienylalanine did not inhibit the growth of $P$. aeruginosa on minimal agar plates with glucose as the carbon source but with fructose there was pronounced growth inhibition and resistant colonies were isolated from the inhibition zones after a few days of incubation. Calhoun \& Jensen (1972) suggest that the metabolic pool of cells growing on fructose is relatively low in the precursors for the synthesis of aromatic amino acids so that analogues of these acids are able to compete successfully for certain enzymes and exert a growth inhibitory effect. These results suggest that if growth media can be devised which decrease the metabolic pools of precursors of other amino acids it might be possible to increase the sensitivity of P. aeruginosa to metabolic analogues and thereby isolate regulatory mutants. Leisinger, Haas \& Hegarty (1972) found that $P$. aeruginosa was more sensitive to growth inhibition by indospicine (an analogue of arginine) when the cultures were grown on an ornithine medium, than in a glucose + arginine medium. With $2 \mathrm{~mm}$-indospicine the doubling time in ornithine medium was increased eightfold from the control culture while in the glucose + arginine medium the increase was only twofold. With 2 mm-canavanine the mean generation time was increased twofold in both media.

For catabolic enzymes it could be predicted that if a substrate analogue could give rise to a toxic product it should be possible to increase the sensitivity of the culture to the analogue by arranging growth conditions so that the bacteria contained a high level of the enzyme concerned. Hynes \& Pateman (1970) found that mutants of Aspergillus nidulans which produced acetamidase constitutively were more sensitive to growth inhibition by fluoracetamide than was the wild-type strain. They were able to isolate acetamide-negative mutants from a semiconstitutive mutant plated on an acetate medium containing 2 mg fluorace. tamide/ml after treatment of a conidial suspension with $N$-methyl- $N^{\prime}$-nitro- $N$-nitrosoguanidine. We have adapted this method to enable us to isolate amidase-negative mutants of Pseudomonas aeruginosa by using growth media which allow the maximum rate of amidase synthesis by the parental strains.

Pseudomonas aeruginosa PACI produces an aliphatic amidase which is induced by acetamide and propionamide and allows these two amides to be used as the carbon and nitrogen sources for growth. Constitutive mutants have been isolated on $\mathrm{S} / \mathrm{F}$ plates containing sodium succinate $(\mathrm{I} \%)$ and formamide $(0.1 \%)$. Formamide is a poor substrate and very 
Table I. Effect of fluoroacetamide on growth of Pseudomonas aeruginosa

Cultures grown in $5 \mathrm{ml}$ minimal salt medium with succinate or pyruvate as the carbon source for growth, inoculated with $0.05 \mathrm{ml}$ of a $24 \mathrm{~h}$ culture grown in the same medium, with the exception of the induced wild-type strain which was grown in minimal medium containing $0.5 \%$ lactamide. Fluoroacetamide was added at various concentrations and the cultures incubated for $24 \mathrm{~h}$ at $37^{\circ} \mathrm{C}$ with shaking.

\begin{tabular}{|c|c|c|c|c|c|c|c|c|}
\hline \multirow[b]{2}{*}{ Strain } & \multirow[b]{2}{*}{ Carbon source } & \multicolumn{7}{|c|}{ Fluoroacetamide added $(\mathrm{mg} / \mathrm{ml})$} \\
\hline & & o & 0.4 & 0.8 & $1 \cdot 0$ & $2 \cdot 0$ & $4 \cdot 0$ & 10 \\
\hline \multicolumn{9}{|l|}{ PACI } \\
\hline Wild-type & Succinate & ++ & + & + & + & + & + & - \\
\hline Non-induced & Pyruvate & ++ & + & + & + & $\operatorname{tr}$ & - & - \\
\hline \multirow[t]{2}{*}{ Induced } & Succinate & ++ & + & + & + & + & + & - \\
\hline & Pyruvate & ++ & $\operatorname{tr}$ & $\operatorname{tr}$ & - & - & - & - \\
\hline \multicolumn{9}{|l|}{ PAC307 } \\
\hline Amidase- & Succinate & ++ & ++ & ++ & ++ & ++ & ++ & ++ \\
\hline negative & Pyruvate & $+t$ & ++ & $+t$ & ++ & ++ & $t+$ & ++ \\
\hline \multicolumn{9}{|l|}{ PACIII } \\
\hline \multirow[t]{2}{*}{ Constitutive } & Succinate & ++ & + & + & + & + & + & - \\
\hline & Pyruvate & ++ & - & - & - & - & - & - \\
\hline \multicolumn{9}{|l|}{ PACI 42} \\
\hline Constitutive & Succinate & ++ & + & + & + & + & - & - \\
\hline $\begin{array}{l}\text { Catabolite } \\
\text { repression- } \\
\text { resistant }\end{array}$ & Pyruvate & ++ & - & - & - & - & - & - \\
\hline
\end{tabular}

Growth expressed as equivalent to $E_{870 \mathrm{~nm}}$ as follows:,$++>\mathrm{I} \cdot 0 ;+, 0.5$ to $\mathrm{I} \cdot 0 ;$ tr, barely visible; ,- no growth detected.

poor inducer and the wild-type inducible strain grows feebly on S/F plates whereas constitutive mutants grow rapidly and produce large colonies (Brammar, Clarke \& Skinner, 1967). The enzyme is also very sensitive to catabolite repression by succinate and mutants which are resistant to catabolite repression have been isolated on $\mathrm{S} / \mathrm{L}$ plates containing sodium succinate $(\mathrm{I} \%)$ and lactamide $(0.02 \%)$. Succinate is a very effective catabolite repressor and lactamide is a good inducer but a very poor substrate (Clarke, I970). Amidasenegative mutants have been isolated as shadowy colonies on acetamide plates supplemented with traces of succinate (Skinner \& Clarke, 1968).

Preliminary tests with wild-type and constitutive strains indicated that Pseudomonas aeruginosa was not very sensitive to growth inhibition by fluoroacetamide. However, a strain which was both constitutive and resistant to catabolite repression, PACI42, was inhibited by $5 \mathrm{mg}$ fluoroacetamide/ $\mathrm{ml}$ on minimal agar plates containing sodium acetate $(0.3 \%)$ as carbon source or $2 \mathrm{mg}$ fluoroacetamide $/ \mathrm{ml}$ with sodium pyruvate (I \%) as carbon source. The plates were spread with about $10^{9}$ bacteria suspended in dilution buffer from an overnight culture in nutrient broth. For some experiments a crystal of $\mathrm{N}$ methyl- $N^{\prime}$-nitro- $N$-nitrosoguanidine was placed in the centre of the plate to induce mutagenesis. After 2 or 3 days of incubation at $37^{\circ} \mathrm{C}$ fluoroacetamide-resistant colonies appeared. These were picked off and streaked out on fluoroacetamide/pyruvate plates and subsequently plated out on the same medium to obtain single colonies of the mutants free from background parental bacteria. The fluoroacetamide-resistant mutants were of several classes. Some mutants grew slightly on acetamide and not at all on lactamide and were presumed to produce a defective amidase; others were acetamide-negative and were presumed to have defects in the amidase structural gene or a controlling element; others grew at near normal 
rates on acetamide and acetate and were presumed to have mutations in amidase regulator genes; no acetate-negative mutants were recovered by this method. In some experiments spontaneous fluoroacetamide-resistant mutants were selected and these all appeared to be amidase-negative and several had zero reversion rates and were thought likely to be"deletion mutants.

The method was adapted for the isolation of amidase-negative mutants from other strains. The constitutive strain PACII I was grown overnight in pyruvate medium to get maximum derepression of the bacteria used as the inoculum and the best results for mutant isolation were obtained with plates containing $2 \mathrm{mg}$ fluoroacetamide/ $\mathrm{ml}$ and $0.5 \%$ of sodium pyruvate. The wild-type strains PACI and PAOI (Holloway, 1955) were grown overnight in minimal medium containing $0.5 \%$ lactamide to induce amidase synthesis to a high level. Temperature-sensitive mutants were isolated from plates incubated for 2 days at $30^{\circ} \mathrm{C}$ which were then transferred to $37^{\circ} \mathrm{C}$ and re-incubated overnight. The colonies which appeared after the second incubation at $37^{\circ} \mathrm{C}$ were the temperature-sensitive amidasenegative mutants.

To isolate amidase-negative mutants of Pseudomonas putida A87, the wild-type strain was first grown on S/F plates to obtain a constitutive mutant. This was then used to isolate acetamide-negative mutants from fluoroacetamide + pyruvate plates. It was not possible to isolate constitutive mutants from $P$. cepacia 716 but the wild-type inducible strain was successfully used to isolate acetamide-negative mutants by growing the inoculum in $0.5 \%$ lactamide medium, as for the wild-type strain of P. aeruginosa (Betz \& Clarke, 1973).

The effect of the carbon source on the amidase activities of the various strains and the response to fluoroacetamide could be seen very clearly when the cultures were grown in liquid medium (Table I). The amidase-negative mutant PAC307 was completely resistant to fluoroacetamide in both succinate and pyruvate medium up to concentrations of $10 \mathrm{mg} / \mathrm{ml}$. The wild-type PACI, non-induced, grew quite well in succinate medium with up to $4 \mathrm{mg}$ fluoroacetamide/ml but growth was markedly inhibited in pyruvate medium at $2 \mathrm{mg} / \mathrm{ml}$ and the induced culture was inhibited at $0.4 \mathrm{mg} / \mathrm{ml}$. Although on plates it had been much easier to devise a fluoroacetamide growth-inhibitory medium for the catabolite repressionresistant strain PACI42, the difference between this strain and the constitutive strain PACI I I was not so marked in liquid medium. However, growth in succinate medium of PACI 42 was inhibited by $4 \mathrm{mg}$ fluoroacetamide/ml while that of PACIII was inhibited at $10 \mathrm{mg} / \mathrm{ml}$ but not at the lower concentrations used.

The resistance of Pseudomonas aeruginosa to analogues of other metabolites of both biosynthetic and catabolic pathways may be altered by changing the environmental conditions to allow maximum or minimum rates of synthesis of enzymes which might make it possible to devise positive selection methods for regulatory and structural gene mutants.

\section{REFERENCES}

Betz, J. L. \& ClaRke, P. H. (1973). Growth of Pseudomonas species on phenylacetamide. Journal of General Microbiology 75, I67-1 77.

Brammar, W. J., Clarke, P. H. \& Skinner, A. J. (1967). Biochemical and genetic studies with regulator mutants of the Pseudomonas aeruginosa 8602 amidase system. Journal of General Microbiology 47, 87102.

CALHoun, D. H. \& J JenSEN, R. A. (1972). Significance of altered carbon flow in aromatic amino acid synthesis: an approach to the isolation of regulatory mutants in Pseudomonas aeruginosa. Journal of Bacteriology 109, $365-372$.

Clarke, P. H. (1970). The aliphatic amidases of Pseudomonas aeruginosa. Advances in Microbial Physiology 4, $179-222$. 
Holloway, B. W. (1955). Genetic recombination in Pseudomonas aeruginosa. Journal of General Microbiology 13, 572-581.

Holloway, B. W. (1969). Genetics of Pseudomonas. Bacteriological Reviews 33, 419-443.

Hynes, M. J. \& Pateman, J. A. J. (1970). The genetic analysis of regulation of amidase synthesis in Aspergillus nidulans. II. Mutants resistant to fluoroacetamide. Molecular and General Genetics 108, 107-I I6.

LeISINGER, T., HAAS, D. \& Hegarty, M. P. (1972). Indospicine as an arginine antagonist in Escherichia coli and Pseudomonas aeruginosa. Biochimica et biophysica acta 262, 21 4-219.

Skinner, A. J. \& Clarke, P. H. (1968). Acetate and acetamide mutants of Pseudomonas aeruginosa 8602. Journal of General Microbiology 50, 183-194. 